\title{
Activated EphA2 Processing by MT1-MMP Is Involved in Malignant Transformation of Ovarian Tumours In Vivo
}

\author{
YOKO TAKAHASHI ${ }^{1,2}$, MAKOTO HAMASAKI ${ }^{1}$, MIKIKO AOKI $^{1}$, KAORI KOGA $^{1}$, \\ NAOHIKO KOSHIKAWA ${ }^{3}$, SHINGO MIYAMOTO ${ }^{2}$ and KAZUKI NABESHIMA ${ }^{1}$ \\ ${ }^{1}$ Department of Pathology, Faculty of Medicine, Fukuoka University, Fukuoka, Japan; \\ ${ }^{2}$ Department of Obstetrics and Gynecology, Faculty of Medicine, Fukuoka University, Fukuoka, Japan; \\ ${ }^{3}$ Division of Cancer Cell Research, Kanagawa Cancer Center Research Institute, Kanagawa, Japan
}

\begin{abstract}
Background/Aim: Erythropoietin-producing hepatocellular receptor-2 (EphA2) is overexpressed in ovarian cancer. The $N$-terminals of EphA2 are processed by membrane-type 1 matrix metalloproteinase (MT1-MMP) and can subsequently induce ligand-independent signal activation to promote motility, invasion, and metastasis. The aim of this study was to investigate whether EphA2 processing occurs in benign, borderline, and malignant ovarian tumours. Materials and Methods: Overall 107 ovarian epithelial carcinomas (OECs; 47 serous, 24 endometrioid, 16 mucinous, and 20 clear cell), 54 ovarian borderline tumours (OBTs; 12 serous, 42 mucinous), and 45 adenomas (15 serous, 17 mucinous, and 13 endometriotic cysts) were evaluated. Expression and processing of EphA2 were semi-quantitatively analyzed. EphA2 processing was also investigated by immunoblotting. Results: EphA2 and MT1-MMP co-expression were detected. $\mathrm{N}$ terminal EphA2 levels were significantly lower than those of $C$-terminal EphA2 in OECs and OBTs, but not in adenomas. Immunoblotting revealed processed fragments in OEC and OBTs. Conclusion: EphA2 processing by MT1-MMP is associated with malignant transformation in ovarian tumours.
\end{abstract}

Ovarian cancer is the most common cause of death from any gynaecological disease and, to date, there is no effective screening method for its early diagnosis. Many cases of ovarian cancer are diagnosed at an advanced stage due to either peritoneal fluid or metastasis $(1,2)$. Therefore, it is important to understand the molecular mechanism of ovarian tumours, to apply this information to the development of new biomarkers or therapeutic strategies, and thus improve the poor prognosis.

Correspondence to: Kazuki Nabeshima, MD, Ph.D., Department of Pathology, Fukuoka University Faculty of Medicine, Nanakuma $7-$ 45-1, Jonan ward, Fukuoka, Japan. Tel: +81 928011011, Fax: +81 928659901, e-mail: kaznabes@fukuoka-u.ac.jp

Key Words: EphA2, MT1-MMP, processing, ovarian tumours.
EphA2 (erythropoietin-producing hepatocellular receptor2) is a member of the erythropoietin-producing human hepatoma (EPH) family of receptor tyrosine kinases, which interact with cell-bound ligands known as ephrins. Whereas the specific role of EphA2 in normal epithelium is not completely understood, potential roles for EphA2 in tumourigenesis include regulation of cell growth, survival, invasion, and angiogenesis (2). In normal cells, EphA2 autophosphorylates with EphrinA1 and inhibits Ras that has been activated by ErbB-receptor signals. In contrast, overexpression of EphA2 in cancer cells promotes invasion and metastasis in a ligand-independent manner (3). It is reported that EphA2 is overexpressed in some malignant tumours, such as lung (4), breast, ovary $(2,5,6)$, prostate (7), colon (8), and skin (3). In ovarian cancer, EphA2 overexpression has been reported as predictive of aggressive development $(5,6)$ associated with angiogenesis (2). Recently the ligand-binding domain of EphA2, which resides in the N-terminal portion of the molecule, has been shown to be cleaved by MT1-MMP, activating an oncogenic signal that results in enhanced cancer cell motility and invasion, even though soluble Ephrin-A1 is abundantly expressed (9).

To our knowledge, there are no published reports evaluating the role of EphA2 expression in ovarian tumours, particularly with respect to EphA2 processing. Here, we compared the processing of EphA2 in ovarian cancers, borderline tumours, and benign tumours using immunostaining and Western blot analyses. Moreover, mediation of EphA2 processing by MT1MMP was evaluated through the use of an in situ proximity ligation assay (PLA).

\section{Materials and Methods}

Tissue samples. This study included ovarian tumour tissue samples from 206 patients, which were obtained from ovarian tumour files at the Department of Pathology, Fukuoka University Hospital. The patients were clinically treated at the Department of Gynecologic Oncology, Fukuoka University Hospital between January 2000 and December 2014. Samples of ovarian epithelial tumours included 45 
benign ovarian tumours ( 15 serous cyst adenomas, 17 mucinous cyst adenomas, and 13 endometrial cysts), 54 ovarian borderline tumours (OBTs; 12 serous borderline tumours, and 42 mucinous borderline tumours), and 107 ovarian carcinomas (47 serous adenocarcinomas, 24 endometrioid carcinomas, 16 mucinous carcinomas, and 20 clear cell carcinomas). Tumor tissue specimens for western blot analyses were frozen in liquid nitrogen and kept at $-80^{\circ} \mathrm{C}$ until use.

Immunohistochemistry. Surgically resected specimens were fixed in $10 \%$ formalin and processed into paraffin blocks. Tissues were sectioned (3- $\mu \mathrm{m}$ thickness), and the sections were deparaffinized and immersed in $0.3 \%$ hydrogen peroxide in methanol for $10 \mathrm{~min}$ at room temperature (RT) to block endogenous peroxidase activity and then heated in $10 \mathrm{mM}$ citrated buffer ( $\mathrm{pH} 6.0)$ in a microwave oven $(700$ W) for $10 \mathrm{~min}$ to retrieve epitopes. After non-specific sites were blocked with $5 \%$ non-fat dry milk for $1 \mathrm{~h}$ at RT, these sections were incubated overnight at $4{ }^{\circ} \mathrm{C}$ with polyclonal antibodies against the Cterminal of EphA2 (C-EphA2) (1:200; Santa Cruz Biotechnology Inc., Santa Cruz, CA, USA) (9), the N-terminal of EphA2 (N-EphA2) (1:800; Abgent, San Diego, CA, USA) (10), or monoclonal antibodies against MT1-MMP (1:50; 2D12) (11). Subsequently, these sections were washed and incubated with ChemMate EnVision (Dako, Carpinteria, CA, USA). Immunoreacitve proteins were visualized with 3, 3'-diaminobenzidine (DAB; Dako), followed by counterstaining with haematoxylin. In all cases skin sections were stained to represent the normal control on the same glass slide in order to elucidate the staining pattern and intensity in normal epidermis (3).

Immunohistochemical results were assessed as described in previous reports $(3,9)$. Similar expression levels of N-EphA2 and $\mathrm{C}$-EphA2 indicated that the EphA2 likely retained the N-terminal ligand-binding domain. In contrast, reduced expression levels of $\mathrm{N}$ EphA2 compared with the C-terminal indicated that N-EphA2 was likely to have been cleaved off.

Visual- and computer-supported evaluation of immunohistochemical staining. Immunohistochemical expression of C-EphA2, N-EphA2, and MT1-MMP in all samples was assessed using Tissue Studio v.2.0 software (Definiens AG, Munich, Germany). For image analysis, each immunohistochemically stained slide was scanned and converted to a whole-slide image (WSI, also known as a virtual slide) with NanoZoomer 2.0-RS (Hamamatsu Photonics, Hamamatsu, Japan) at $20 \times$ magnification. On each WSI, the tumour area was selected by a hand-drawing tool, and tumour cells exhibiting cytoplasmic expression of EphA2 and MT1-MMP were identified using Tissue Studio v.2.0, installed on the server for NanoZoomer 2.0-R.S. The DAB colour intensity of positive tumour cells in every unit area was measured, and the average value per unit area $\left(/ \mu \mathrm{m}^{2}\right)$ was calculated using Tissue Studio v.2.0. The intensity of EphA2 and MT1-MMP staining was measured in epithelial cells of normal skin, which had been added as a positive control to each glass slide alongside tumour tissues. Furthermore, the ratio of the average staining intensity of tumour cells to that of the control epithelial cells was calculated.

Protein extraction and western blot analysis. Proteins were extracted from frozen tissues in RIPA lysis buffer (50 mM Tris-HCl, ph7.4, 150 $\mathrm{mM} \mathrm{NaCl}, 1$ mM EDTA, 1\% NP-40; Millipore, Bedford, MA, USA), containing protease inhibitors (Complete Mini; Roche Applied Sciences, Penzberg, Germany) using a homogenizer on ice. The extracts were clarified by centrifugation $(14,000 \mathrm{rpm}$ for $20 \mathrm{~min}$ at
Table I. Characteristics of ovarian epithelial carcinomas (OECs).

\begin{tabular}{|c|c|c|}
\hline & $\mathrm{N}$ & $\%$ \\
\hline Patients & 107 & \\
\hline \multicolumn{3}{|l|}{ Age (years) } \\
\hline Mean & 55 & \\
\hline Range & $31-86$ & \\
\hline \multicolumn{3}{|l|}{ FIGO stage } \\
\hline I & 51 & 47.6 \\
\hline II & 14 & 13.0 \\
\hline III & 26 & 24.2 \\
\hline IV & 14 & 13.0 \\
\hline Unknown & 2 & 1.8 \\
\hline \multicolumn{3}{|l|}{ Histology } \\
\hline Serous & 47 & 43.9 \\
\hline Endometrioid & 24 & 22.4 \\
\hline Mucinous & 16 & 15.0 \\
\hline Clear cell & 20 & 18.7 \\
\hline \multicolumn{3}{|l|}{ Grade } \\
\hline 1 & 47 & 43.9 \\
\hline 2 & 35 & 32.7 \\
\hline 3 & 25 & 23.3 \\
\hline \multicolumn{3}{|c|}{ Ascites (cytology) } \\
\hline Negative & 52 & \\
\hline Positive & 52 & \\
\hline Unknown & 3 & \\
\hline \multicolumn{3}{|c|}{ LN metastasis $(n=85)$} \\
\hline Negative & 67 & \\
\hline Positive & 18 & \\
\hline \multicolumn{3}{|l|}{ Recurrence } \\
\hline No & 52 & \\
\hline Yes & 41 & \\
\hline
\end{tabular}

$4^{\circ} \mathrm{C}$ ) and subjected to sodium dodecyl sulfate-polyacrylamide gel electrophoresis (SDS-PAGE). The proteins were then transferred electrophoretically to Immobilon membranes (Millipore). Nonspecific sites were blocked with 5\% dry non-fat milk in TBS at $37^{\circ} \mathrm{C}$ for $1 \mathrm{~h}$, and then the membrane was incubated overnight at $4^{\circ} \mathrm{C}$ with antiEphA2 (Santa Cruz Biotechnology) (9) and anti-MT1-MMP (2D12) (11) antibodies. After three washes with TBS-T (TBS containing $0.05 \%$ Tween-20), the membranes were incubated for $1 \mathrm{~h}$ with peroxidase-conjugated anti-rabbit IgG; immunoreactive proteins were detected with chemiluminescence reagents according to the manufacturer's instructions (PerkinElmer, Waltham, MA, USA).

In situ Proximity Ligation Assay (PLA). Duolink PLA (Olink, Uppsala, Sweden), was used to detect protein-protein interactions. This technique utilizes one pair of oligonucleotide labelled antibodies binding in close proximity (30-40 nm apart) to two different proteins in a complex. PLAs were performed according to the manufacturer's instructions. Briefly, sections were pretreated, and primary antibodies against C-EphA2 (1:200; Santa Cruz Biotechnology) (9), N-EphA2 (1:800; Abgent) (10), and MT1-MMP $(1: 50 ; 2 \mathrm{D} 12)(11)$ were applied for $1 \mathrm{~h}$ at $37^{\circ} \mathrm{C}$. For isotype controls, the primary antibody was substituted with either rabbit (C-EphA2 or N-EphA2) or mouse (MT1-MMP) IgG. Sections were then washed twice for 5 min each in Duolink wash buffer A (Olink) before PLA 


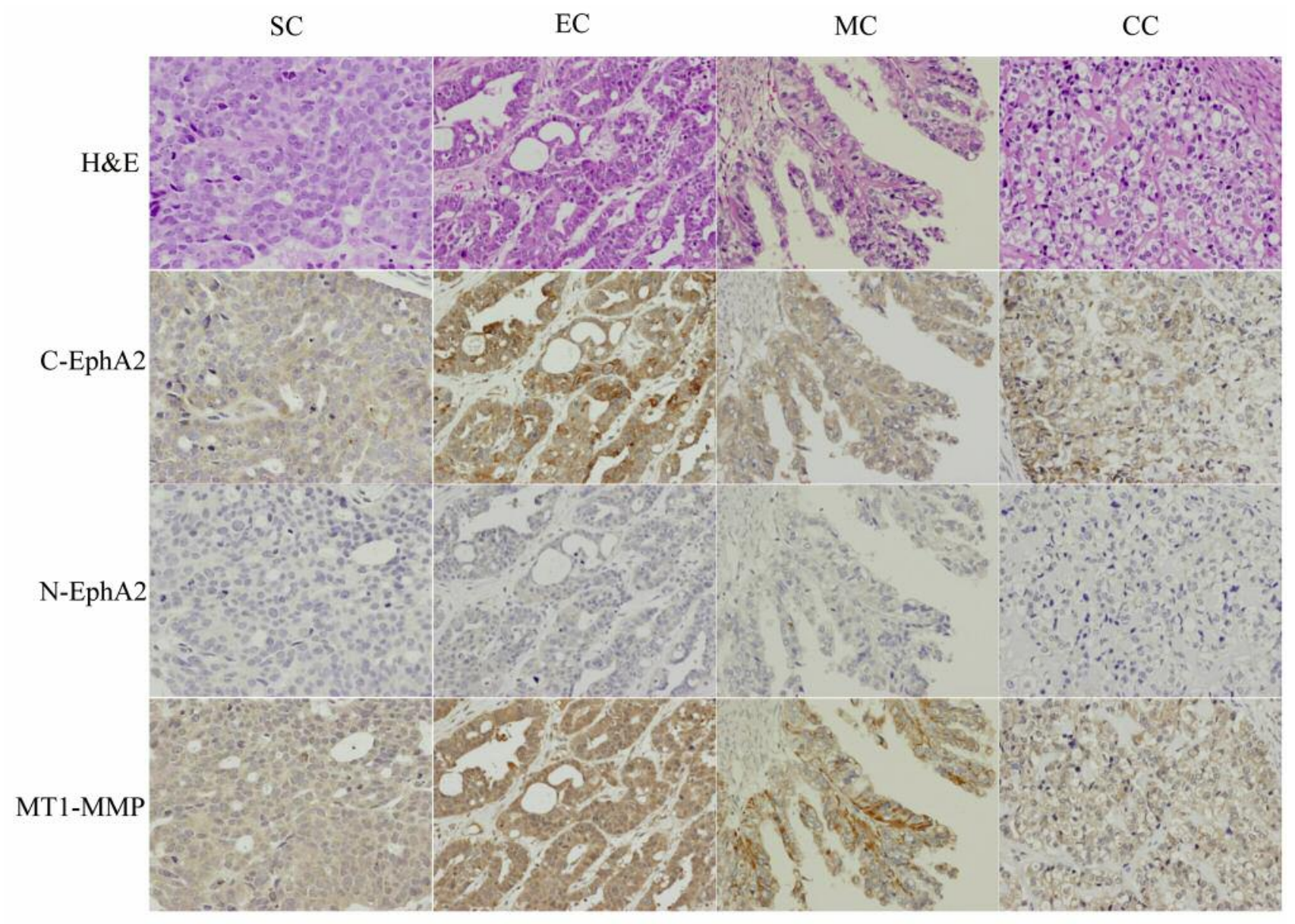

Figure 1. Representative immunohistochemical expression of C-EphA2, N-EphA2, and MT1-MMP in ovarian epithelial carcinomas (OECs; SC: Serous carcinoma; EC: endometrioid carcinoma; MC: mucinous carcinoma; CC: clear cell carcinoma).

PLUS and MINUS probes (Olink) were applied for $1 \mathrm{~h}$ at $37^{\circ} \mathrm{C}$. Following washing (as previously), ligation-ligase solution (Olink) was applied to each sample for $30 \mathrm{~min}$ at $37^{\circ} \mathrm{C}$. Sections were washed again, and amplification- polymerase solution (Olink) was applied for $120 \mathrm{~min}$ at $37^{\circ} \mathrm{C}$. In paraffin sections of formalin-fixed tissues, dot signals were visualized with DAB. The number of in situ PLA signals per cell was determined using Tissue Studio v.2.0.

Compliance with ethical standards. This study was approved by the Ethics Committee of Fukuoka University School of Medicine (No.15-3-14). Use of anonymous and redundant tissue is part of the standard treatment agreement with patients in our hospitals when no objection has been expressed.I

Statistical analyses. Quantitative data are presented as mean \pm standard deviation (SD) and were analysed using the Student's $t$-test. A $p$-value $<0.05$ was considered indicative of statistical significance. All data analyses were conducted using the Excel statistical software package (Ekuseru-Toukei 2015; Social Survey Research Information Co., Ltd., Tokyo, Japan).

\section{Results}

Clinicopathological findings. The clinicopathological characteristics of the 107 cases of ovarian epithelial carcinomas (OECs) are summarized in Table I. The samples included 47 cases of serous carcinoma (SCs, 43.9\%), 24 of endometrioid carcinoma (ECs, 22.4\%), 16 of mucinous carcinoma (MCs, $15.0 \%$ ), and 20 of clear cell carcinoma (CCs, 18.7\%). Fiftyfour cases of OBTs (12 cases of serous and 42 cases of mucinous), 32 cases of adenomas (17 cases of mucinous and 15 cases of serous adenomas) and 13 cases of endometriotic cysts were also included for comparative examination. Although the number of cases of clear cell carcinoma is generally greater than those of endometrioid carcinoma in Japan, the present study was an exception to this; there were more of the latter. In two cases the FIGO stage was missing from the medical chart, thus they were classified as unknown. 


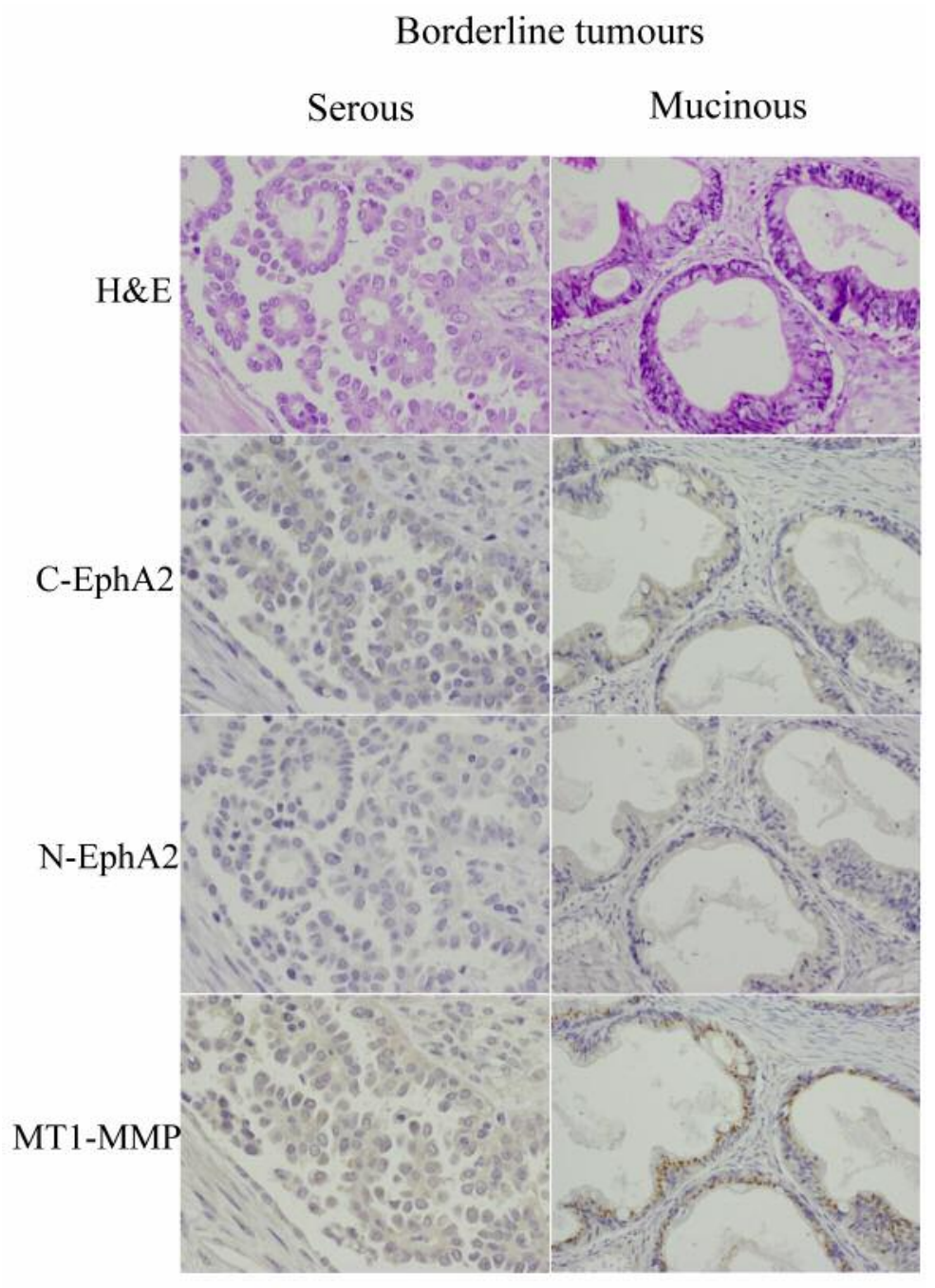

Figure 2. Representative immunohistochemical expression of C-EphA2, N-EphA2, and MT1-MMP in ovarian borderline tumours (OBTs).

Immunohistochemical staining of EphA2 and MT1-MMP. Expression of EphA2 and MT1-MMP was examined immunohistochemically in OECs, OBTs, and benign tumours. Representative examples are shown in Figures 1, 2, and 3. Both C-EphA2 and MT1-MMP were expressed on cell membranes and in the cytoplasm. C-EphA2 was expressed diffusely throughout the tumour in most OEC cases. Interestingly, all histological types of OECs (Figure 4A) and OBTs (Figure 4B) showed significantly lower levels of expression of $\mathrm{N}$ - EphA2 than C-EphA2 $(p<0.001)$. OECs showed a lower expression of N-EphA2 irrespective of the status of FIGO stages, grades, LN metastasis, and ascites cytology (Table II). There was no significant difference of expression between N-EphA2 and C-EphA2 in benign tumours (serous; $p=0.508$, mucinous; $p=0.077$; Figure 4C).
However, expression of EphA2 was rather lower than that of C-EphA2 in endometriotic cyst and in OECs $(p<0.01$; Figure 4C). MT1-MMP was detected in OECs, BOTs, and benign tumours (Figures 1-3).

Detection of EphA2 and MT1-MMP protein in OECs and OBTs by immunoblotting. Expression levels of EphA2 and MT1-MMP proteins were also examined in 10 frozen tissue samples ( 2 serous carcinomas, 1 serous borderline tumour, 2 endometioid carcinomas, 2 mucinous carcinomas, 1 mucinous borderline tumour, and 2 clear cell carcinomas) using immunoblotting. C-EphA2 was expressed as a 130$\mathrm{kDa}$ band, and MT1-MMP appeared as a 65-kDa band (Figure 5). Bands representing processed EphA2 fragments (50-65 kDa) were detected in all samples. 


\section{Benign tumours}

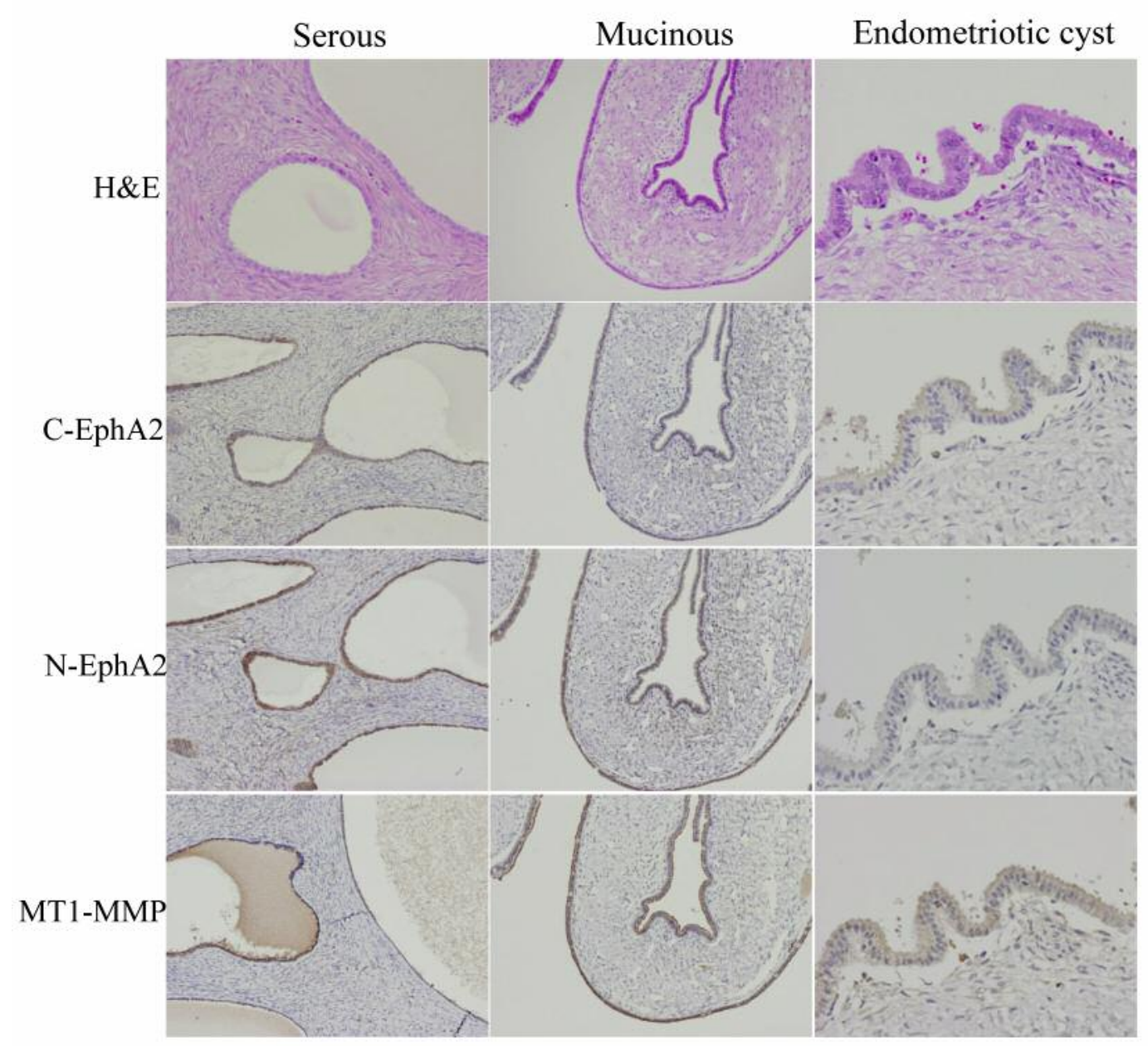

Figure 3. Representative immunohistochemical expression of C-EphA2, N-EphA2, and MT1-MMP in adenomas, and endometriotic cysts.

Expression and interaction of EphA2 and MT1-MMP in serous and mucinous tissues. To examine whether there was interaction between EphA2 and MT1-MMP, we performed in situ PLA using serous and mucinous tumours. In OECs, fewer signals of MT1-MMP and N- EphA2 were observed compared with MT1MMP and C- EphA2 in PLA (serous: $p<0.01$, mucinous: $p=0.039$; Figure 6A and B, carcinoma; Figure 7A and $\mathrm{B}$, carcinoma). In OBTs, fewer signals of MT1-MMP and NEphA2 were also observed compared with MT1-MMP and CEphA2 in PLA, but the differences were not statistically significant (Figure 6A and B, borderline; Figure 7A, B, borderline). On the other hand, there was no significant difference between MT1-MMP and N-EphA2 interaction and that of MT1-MMP and C-EphA2 in adenomas (serous: $p=0.907$, mucinous: $p=0.147$; Figure $6 \mathrm{~A}$ and $\mathrm{B}$, adenoma; Figure 7A and B, adenoma).

\section{Discussion}

To the best of our knowledge, this is the first report to address the possibility that MT1-MMP processing of EphA2 occurs in association with malignant transformation in vivo in human ovarian tumours.

EphA2 retains the ligand-dependent function of Ras inactivation and inhibition of the EGF-EGFR signalling pathway via p120Ras-GAP (12-14). Koshikawa et al. (9) report that the processing of EphA2 by MT1-MMP causes a ligand-independent biological activation in vitro using cell lines or using animal models. Ligand- independent EphA2 activation causes decreased inhibition of Ras/Erk1/2 and the PI3K/Akt signalling pathway via ErbB-receptor $(9,14)$. In addition, phosphorylation of EphA2 at cytoplasmic serine residues induces RhoG/ELMO-2/DOCK-4/Rac-1 signalling, 
A Expression of C-EphA2 and N-EphA2 in OECs

- histological types -

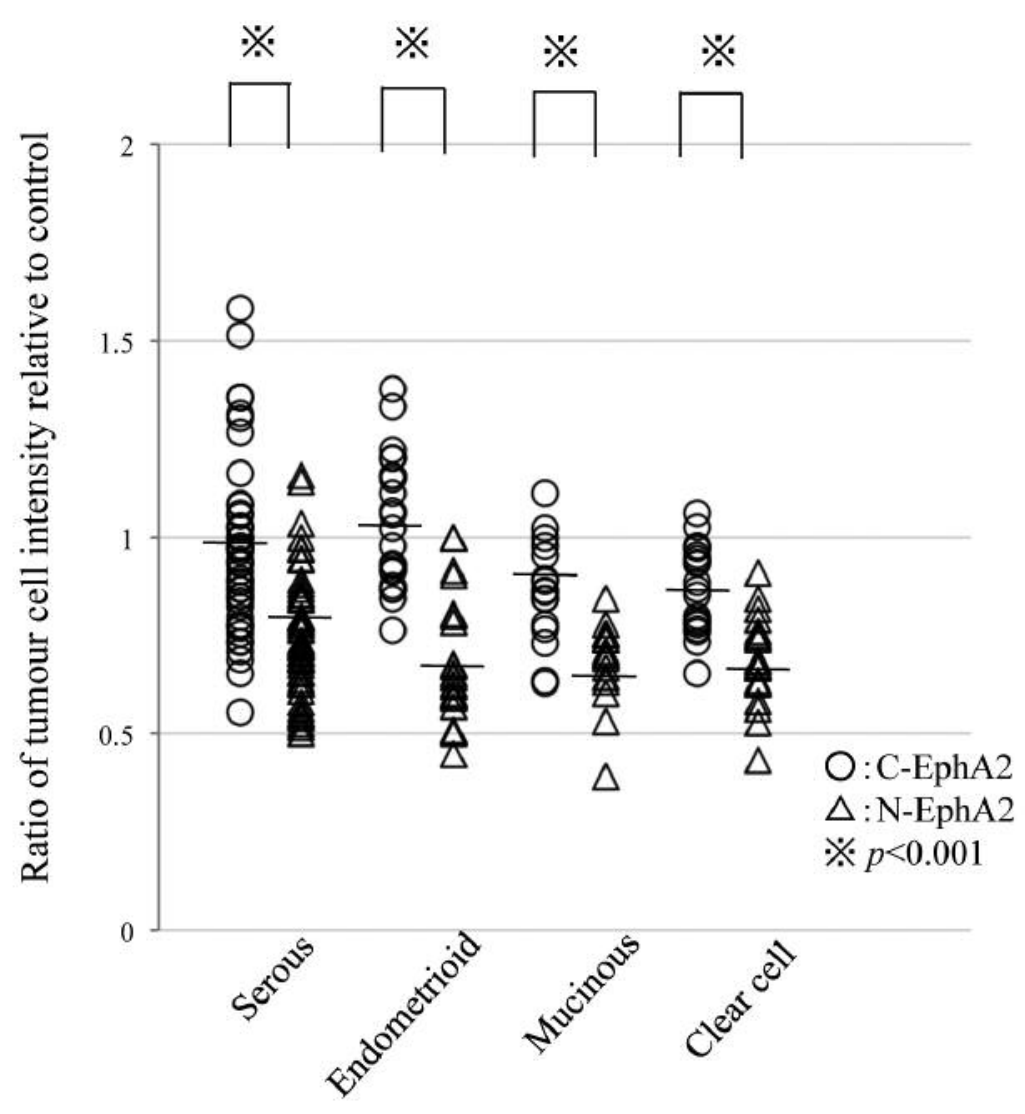

B Expression of C-EphA2 and N-EphA2 in borderline malignancy

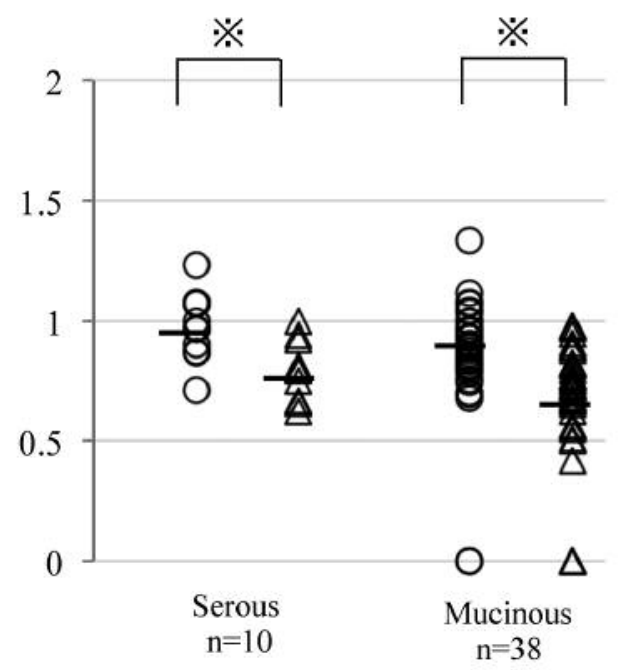

C Expression of C-EphA2 and N- EphA2 in adenomas

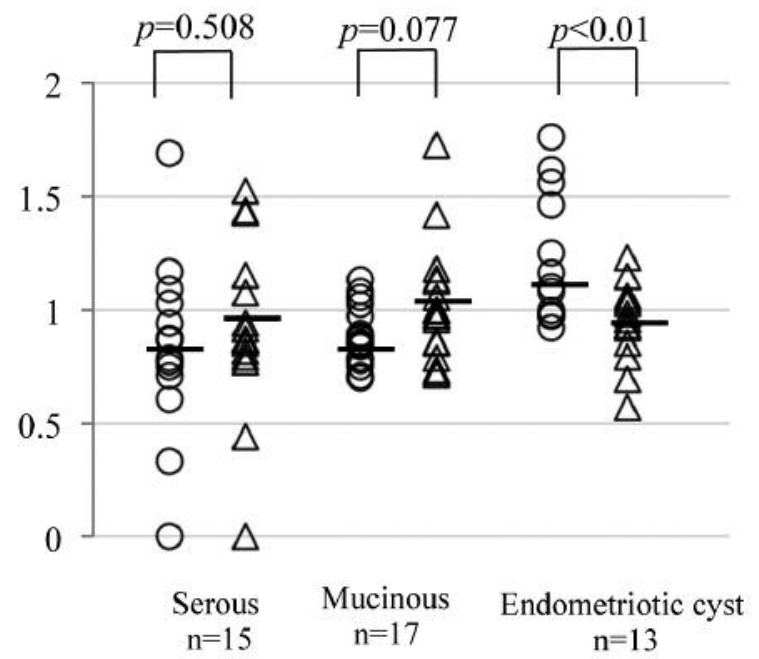

Figure 4. Semiquantitative analysis of C-EphA2 and N-EphA2 in ovarian epithelial carcinomas (OECs) (A), ovarian borderline tumours (OBTs) $(B)$, and adenomas $(C)$ using Tissue Studio v.2.0. Data are mean $\pm S D$. ${ }^{*} p<0.001$ by the Student's $t$-test. 


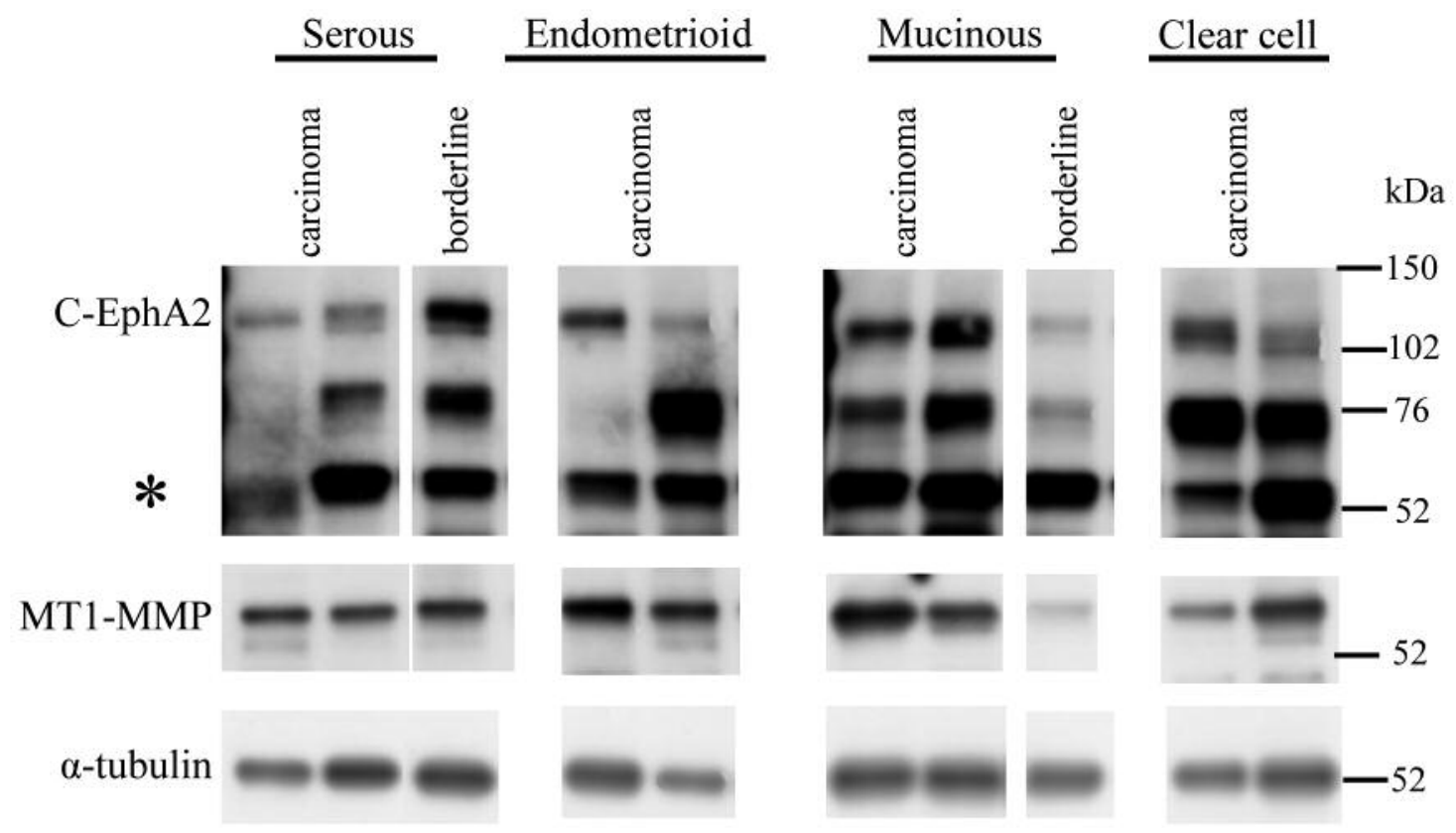

Figure 5. Immunoblotting detection of C-EphA2 and MT1-MMP in tissue extracts from ovarian epithelial carcinomas (OECs). Asterisk shows proteolytic fragments of EphA2 (65- and 60-kDa).

which leads to increased cell migration and invasiveness $(13$, $15,16)$. We investigated whether this processing is also shown in vivo in human ovarian tumour tissues including OECs and OBTs compared with benign lesions such as adenomas and endometriotic cysts. The cleaving and processing of EphA2 was particularly evident in carcinomas and borderline tumours, and EphA2 fragments were also detected by immunoblotting in both tumour types. Moreover, protein-protein interactions were also demonstrated between EphA2 and MT1-MMP using in situ PLA. Co-localization of MT1-MMP and N-EphA2 was significantly reduced compared with co-localization of MT1-MMP and C-EphA2 in OECs, indicative of cleavage of the $\mathrm{N}$-terminal portion of EphA2. OBTs also tended to show a similar reduction of colocalized MT1-MMP and N-EphA2 compared with colocalized MT1-MMP and C-EphA2, although the difference was not statistically significant. However, there was no statistical significance of co-localization of MT1-MMP and C- or N-EphA2 in adenomas. These results suggest that MT1-MMP functions as a cleavage protease of EphA2, especially in the early phase of malignant transformation. It may also have the capability of impacting tumour invasiveness in OECs.

Interestingly, despite having benign endometriotic cysts, similar results were obtained in OECs and OBTs; these were characterized by lower levels of N-EphA2 than C-EphA2. Several previous studies report the expression of MMP-2 and
Table II. Correlation between EphA2 expression levels and clinicopathologica parameters.

\begin{tabular}{lccc}
\hline & \multicolumn{3}{c}{ EphA2 } \\
\cline { 2 - 4 } & C-EphA2 & N-EphA2 & $p$-Value \\
\hline FIGO & & & \\
I $(\mathrm{n}=51)$ & $0.94 \pm 0.19$ & $0.69 \pm 0.13$ & $<0.001$ \\
II $(\mathrm{n}=14)$ & $1.05 \pm 0.06$ & $0.75 \pm 0.05$ & $<0.001$ \\
III (n=26) & $0.91 \pm 0.18$ & $0.73 \pm 0.16$ & $<0.001$ \\
IV (n=14) & $0.95 \pm 0.19$ & $0.77 \pm 0.15$ & $<0.001$ \\
Grade & & & \\
1 (n=47) & $0.92 \pm 0.17$ & $0.71 \pm 0.13$ & $<0.001$ \\
2 (n=35) & $0.97 \pm 0.20$ & $0.70 \pm 0.16$ & $<0.001$ \\
3 (n=25) & $0.98 \pm 0.22$ & $0.75 \pm 0.17$ & $<0.001$ \\
Lymph node metastasis & & & \\
Positive (n=18) & $0.91 \pm 0.14$ & $0.75 \pm 0.15$ & $<0.001$ \\
Negative (n=67) & $0.94 \pm 0.20$ & $0.70 \pm 0.15$ & $<0.001$ \\
Ascites cytology & & & \\
Positive (n=52) & $0.94 \pm 0.17$ & $0.75 \pm 0.15$ & $<0.001$ \\
Negative (n=67) & $0.94 \pm 0.22$ & $0.69 \pm 0.14$ & $<0.001$ \\
\hline
\end{tabular}

C-EphA2: C-terminal of EphA2; N-EphA2: N-terminal of EphA2; aStudent's $t$-test.

MMP-9 in endometriosis $(17,18)$. In addition, endometriosis tissue has been reported to display a high expression of MT1MMP (19), which also activates MMP-2 and induces the migration of endometrial cells. Therefore, our results, showing 


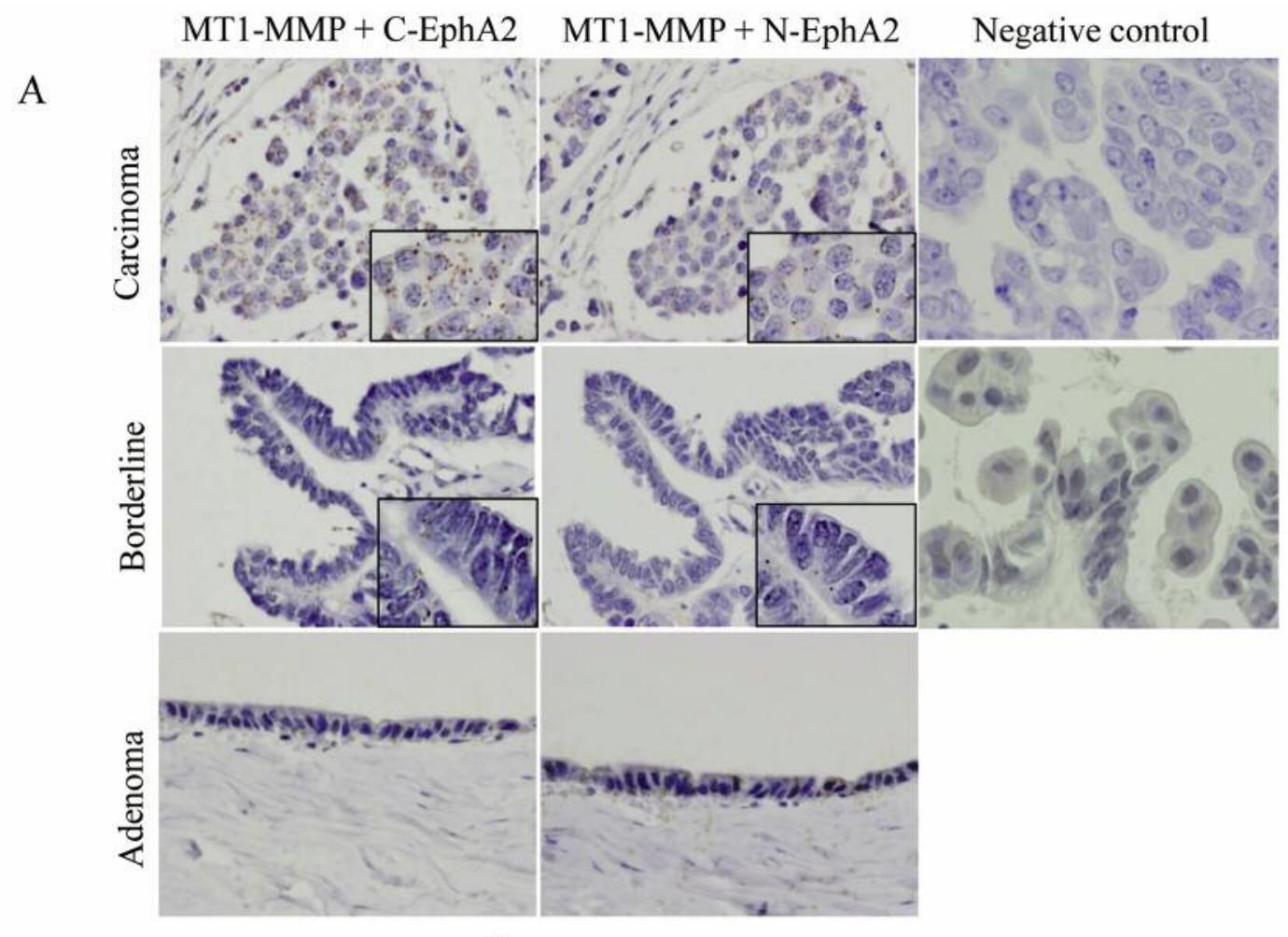

\section{Serous}

B

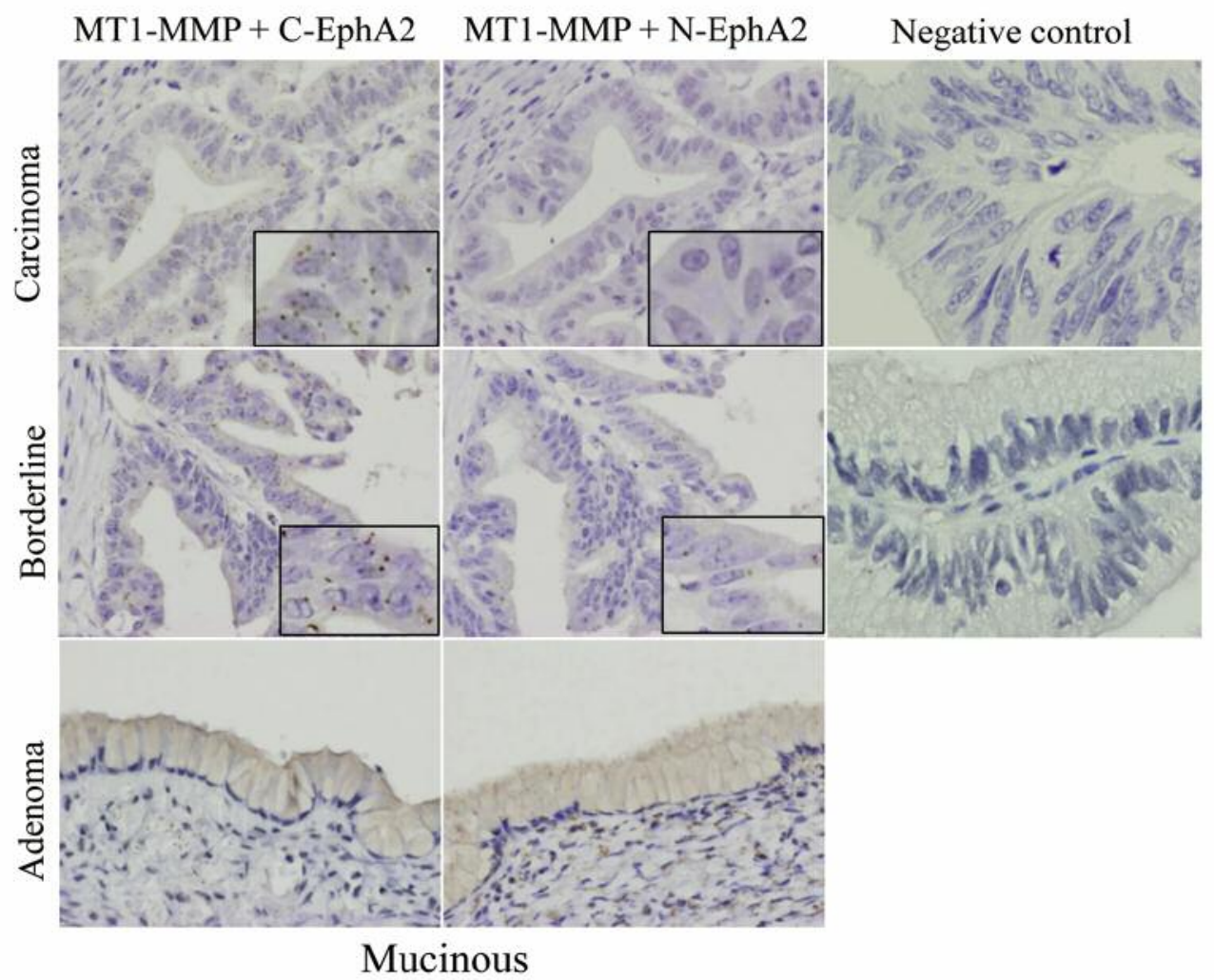

Figure 6. In situ PLA of MT1-MMP and EphA2 expression in ovarian epithelial carcinomas (OECs), ovarian borderline tumours (OBTs), and benign tumours (A: serous, B: mucinous). Visualization by DAB staining. Dots show signals indicative of proximity between MT1-MMP and EphA2. 


\section{$\underline{\text { Serous }}$}

A

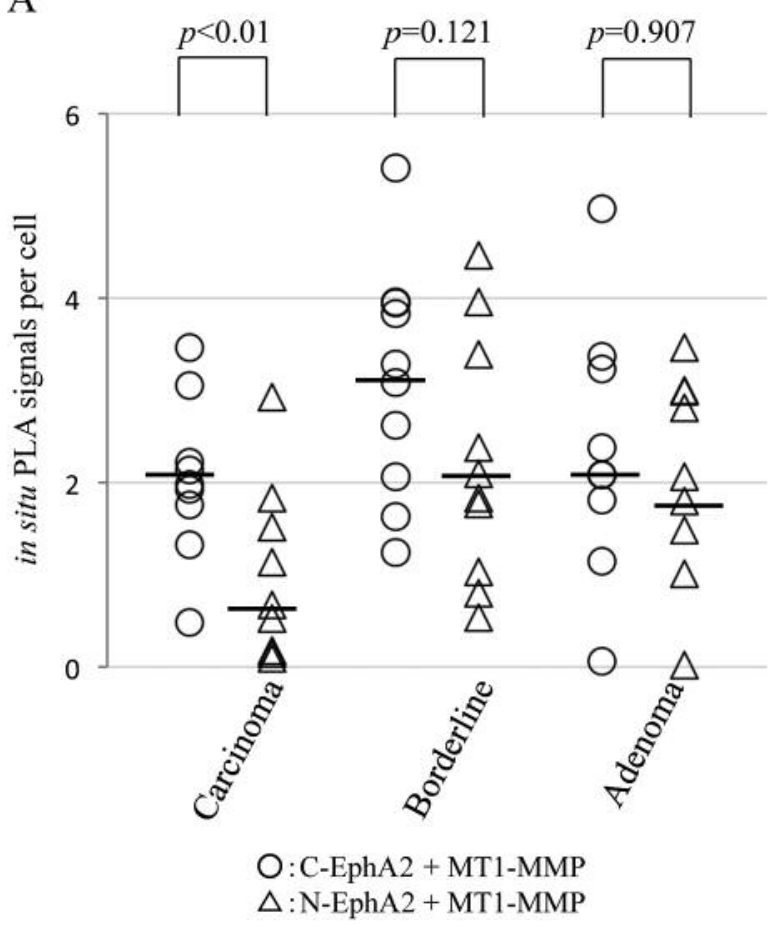

B

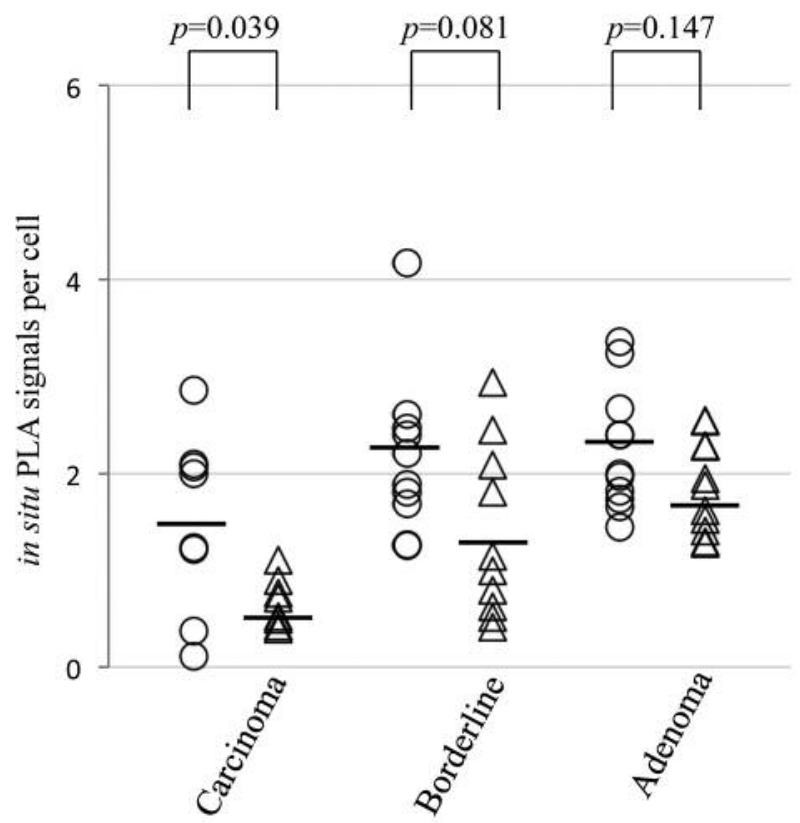

Figure 7. Semiquantitative analysis of the number of MT1-MMP and EphA2 PLA signals per cell in ovarian epithelial carcinomas (OECs; A: serous, B: mucinous). a significant difference in expression between $\mathrm{N}$ - and $\mathrm{C}$ EphA2 and thus processing of EphA2 in endometriotic cyst, are different from the results found in other benign tumours.

EphA2 inhibitors have been developed and supplied clinically; however, their effect are limited and not necessarily satisfactory. Ligand-dependent signal pathways of EphA2 regulate tumour cell growth and survival, but ligand-independent signal pathways also regulate tumour cell motility and invasiveness. EphA2 is also reported as one of the key molecules of angiogenesis in vitro and in vivo (20, 21). Dasatinib acts by inhibiting the ligand-dependent signal pathways via suppressing phosphorylation of the receptor. However, a ligand-independent signalling pathway in OECs was identified, suggesting that inhibiting phosphorylation of the receptor only may not provide sufficient therapeutic effect for OEC patients. For therapeutic strategic targeting of EphA2, it is also important to develop a way to inhibit the processing of EphA2 by MT1-MMP. A multilateral approach may be effective for treatments targeting EphA2 against OECs and OBTs.

\section{Conflicts of Interest}

The Authors declare that they have no conflict of interests.

\section{Acknowledgements}

The Authors would like to thank Ms. M. Onitsuka and Ms. H. Fukagawa for excellent technical assistance in immunohistochemical and in vitro studies. This work was supported in part by grants from the Research Center for Advanced Molecular Medicine, Fukuoka University and the Izumo City Supporting Cancer Research Project.

\section{References}

1 Nam SO, Yotsumoto F, Miyata K, Suzaki Y, Yagi H, Odawara T, Manabe S, Ishikawa T, Kuroki M, Mekada E, and Miyamoto S: Pre-clinical study of BK-UM, a novel inhibitor of HB-EGF, for ovarian cancer therapy. Anticancer Res 34: 4615-4620, 2014.

2 Lin YG, Han LY, Kamat AA, Merritt WM, Landen CN, Deavers MT, Fletcher MS, Urbauer DL, Kinch MS, and Sood AK: EphA2 overexpression is associated with angiogenesis in ovarian cancer. Cancer 109: 332-340, 2007.

3 Tatsukawa R, Koga K, Aoki M, Koshikawa N, Imafuku S, Nakayama J, and Nabeshima K: Immunohistochemical demonstration of EphA2 processing by MT1-MMP in invasive cutaneous squamous cell carcinoma. Virchows Arch 469: 25-34, 2016.

4 Brannan JM, Dong W, Prudkin L, Behrens C, Lotan R, Bekele BN, Wistuba I, and Johnson FM: Expression of the receptor tyrosine kinase EphA2 is increased in smokers and predicts poor survival in non-small cell lung cancer. Clin Cancer Res 15: 4423-4430, 2009.

5 Han L, Dong Z, Qiao Y, Kristensen GB, Holm R, Nesland JM, and Suo Z: The clinical significance of EphA2 and Ephrin A-1 in epithelial ovarian carcinomas. Gynecol Oncol 99: 278-286, 2005. 
6 Thaker PH, Deavers M, Celestino J, Thornton A, Fletcher MS, Landen CN, Kinch MS, Kiener PA and Sood AK: EphA2 expression is associated with aggressive features in ovarian carcinoma. Clin Cancer Res 10: 5145-5150, 2004.

7 Taddei ML, Parri M, Angelucci A, Onnis B, Bianchini F, Giannoni E, Raugei G, Calorini L, Rucci N, Teti A, Bologna M, and Chiarugi P: Kinase-dependent and -independent roles of EphA2 in the regulation of prostate cancer invasion and metastasis. Am J Pathol 174: 1492-1503, 2009.

8 Kataoka H, Igarashi H, Kanamori M, Ihara M, Wang JD, Wang YJ, Li ZY, Shimamura T, Kobayashi T, Maruyama K, Nakamura T, Arai H, Kajimura M, Hanai H, Tanaka M and Sugimura H: Correlation of EPHA2 overexpression with high microvessel count in human primary colorectal cancer. Cancer Sci 95: 136$141,2004$.

9 Koshikawa N, Hoshino D, Taniguchi H, Minegishi T, Tomari T, Nam SO, Aoki M, Sueta $T$, Nakagawa T, Miyamoto S, Nabeshima K, Weaver AM and Seiki M: Proteolysis of EphA2 converts it from a tumor suppressor to an oncoprotein. Cancer Res 75: 3327-3339, 2015.

10 Xiaotin H, Jiandon W, Qian S, Haijin F, Xiaoxiang G and Jinghua W: EphA2/CD10/Bcl-6/MUM 1 contributes to subclassification of diffuse large B cell lymphoma. J Int Transl Med 1: 101-106, 2013.

11 Koshikawa N, Mizushima H, Minegishi T, Eguchi F, Yotsumoto F, Nabeshima K, Miyamoto S, Mekada E and Seiki M: Proteolytic activation of heparin-binding EGF-like growth factor by membrane-type matrix metalloproteinase- 1 in ovarian carcinoma cells. Cancer Sci 102: 111-116, 2011.

12 Beauchamp A and Debinski W: Ephs and ephrins in cancer: ephrin-A1 signalling. Semin Cell Dev Biol 23: 109-115, 2012.

13 Lisabeth EM, Falivelli $G$ and Pasquale EB: Eph receptor signaling and ephrins. Cold Spring Harb Perspect Biol 5: pii: a009159, 2013.

14 Pasquale EB: Eph receptors and ephrins in cancer: bidirectional signalling and beyond. Nat Rev Cancer 10: 165-180, 2010.

15 Miao H and Wang B: EphA receptor signaling - complexity and emerging themes. Semin Cell Dev Biol 23: 16-25, 2012.
16 Inokuchi M, Nakagawa M, Baogok N, Takagi Y, Tanioka T, Gokita K, Okuno K and Kojima K: Prognostic significance of high EphA1-4 expression levels in locally advanced gastric cancer. Anticancer Res 38: 1685-1693, 2018.

17 Shaco-Levy R, Sharabi S, Benharroch D, Piura B and SionVardy N: Matrix metalloproteinases 2 and 9, E-cadherin, and beta-catenin expression in endometriosis, low-grade endometrial carcinoma and non-neoplastic eutopic endometrium. Eur J Obstet Gynecol Reprod Biol 139: 226-232, 2008.

18 Weigel MT, Kramer J, Schem C, Wenners A, Alkatout I, Jonat $\mathrm{W}$, Maass $\mathrm{N}$ and Mundhenke C: Differential expression of MMP-2, MMP-9 and PCNA in endometriosis and endometrial carcinoma. Eur J Obstet Gynecol Reprod Biol 160: 74-78, 2012.

19 Ueda M, Yamashita Y, Takehara M, Terai Y, Kumagai K, Ueki K, Kanda K, Yamaguchi H, Akise D, Hung YC and Ueki M: Survivin gene expression in endometriosis. J Clin Endocrinol Metab 87: 3452-3459, 2002.

$20 \mathrm{Chu} \mathrm{M}$ and Zhang C: Inhibition of angiogenesis by leflunomide via targeting the soluble ephrin-A1/EphA2 system in bladder cancer. Sci Rep 8: 1539, 2018.

21 Sainz-Jaspeado M, Huertas-Martinez J, Lagares-Tena L, Martin Liberal J, Mateo-Lozano S, de Alava E, de Torres C, Mora J, Del Muro XG and Tirado OM: EphA2-induced angiogenesis in ewing sarcoma cells works through bFGF production and is dependent on caveolin-1. PLoS One 8: e71449, 2013. 